Rabaska

Revue d'ethnologie de l'Amérique française

\title{
Centre acadien (Université Sainte-Anne)
}

\section{Ramona Blinn et Carmen d'Entremont}

Volume 18, 2020

URI : https://id.erudit.org/iderudit/1072953ar

DOI : https://doi.org/10.7202/1072953ar

Aller au sommaire du numéro

Éditeur(s)

Société québécoise d'ethnologie

ISSN

1703-7433 (imprimé)

1916-7350 (numérique)

Découvrir la revue

Citer ce document

Blinn, R. \& d'Entremont, C. (2020). Centre acadien (Université Sainte-Anne).

Rabaska, 18, 426-428. https://doi.org/10.7202/1072953ar d'utilisation que vous pouvez consulter en ligne.

https://apropos.erudit.org/fr/usagers/politique-dutilisation/ 


\section{Rapports des institutions}

\section{AcAdie}

\section{Centre acadien}

Université Sainte-Anne

Pointe-de-l’Église (Nouvelle-Écosse)

B0W 1M0
Téléphone : (902) 769-2114, poste 7204

Courriel : centre.acadien@usainteanne.ca

Toile : www.usainteanne.ca/centre-acadien

Le Centre acadien $\left(\mathrm{CA}_{\mathrm{A}}\right)$ a pour mission de collectionner, de conserver et de classer tout ce qui favorise l'histoire et la culture de l'Acadie. Le $\mathrm{C}_{\mathrm{A}}$ gère un centre d'archives, appuie les chercheurs dans les diverses étapes de leurs projets et coordonne des activités de recherche en vue de renforcer l'excellence en recherche et en développement à l'Université Sainte-Anne, seul établissement postsecondaire de langue française en Nouvelle-Écosse. L'équipe du $\mathrm{CA}_{\mathrm{A}}$ veille également à mener des initiatives conjointes avec des organismes communautaires dans le but de favoriser l'épanouissement de la culture acadienne. Voici un aperçu des activités et projets de l'année écoulée.

Activités de promotion et de soutien à la recherche

À l'automne, le CA a organisé un 5 à 7 de la recherche afin de faciliter des échanges autour d'enjeux relatifs au bilinguisme. Andrea Burke-Saulnier, professeure et directrice du Département des sciences de l'éducation, Roger Gervais, professeur au Département des sciences humaines, et Stéphane Cyr, directeur général de la Municipalité de Clare, ont animé une discussion fort intéressante sur le sujet. L'activité, animée par Yvette Comeau, étudiante à la maîtrise, a attiré une vingtaine de personnes.

Une conférence publique sur le bataillon acadien $\left(165^{\mathrm{e}}\right)$ de la Première Guerre mondiale, une facette méconnue de l'histoire acadienne, prononcée par Gregory Kennedy, professeur d'histoire et directeur scientifique de l'Institut d'études acadiennes à l'Université de Moncton, a eu lieu le 5 novembre dernier. Le chercheur Kennedy a mis l'accent sur l'expérience de 86 soldats recrutés à la Baie SainteMarie. Le lendemain, il a offert à une douzaine de personnes un atelier intime sur les sources documentaires utilisées dans le cadre de ses recherches. Ces activités, organisées par la Chaire de recherche en études acadiennes et transnationales (CRÉACT, dont le titulaire est Clint Bruce), en partenariat avec le Centre acadien et la Société historique acadienne de la Baie Sainte-Marie, ont été fortement appréciées du public de l'Université et de la communauté de Clare. 
Encore cette année, le CA a coordonné la semaine de la recherche de l'Université Sainte-Anne. Cette $6^{\mathrm{e}}$ édition, qui s'est tenue du 5 au 11 mars sous le thème "La recherche au service du bien commun : perspectives et enjeux ", avait pour objectif de susciter des échanges autour de sujets préoccupants et actuels, tels que l'environnement, la santé, l'économie, l'éducation et les valeurs humaines. La programmation diversifiée a attiré un grand nombre de participants. Un des événements marquants de la semaine a été la conférence d'honneur du jeudi 5 mars, dispensée par Sylvain Charlebois, professeur titulaire à la Faculté de management et en agriculture à l'Université Dalhousie, et directeur scientifique de l'Institut canadien en prospective agroalimentaire. La conférence de Charlebois, qui a brossé un portrait actuel de la recherche dans le secteur agroalimentaire, a suscité beaucoup d'intérêt ; la popularité de sa webdiffusion en est le témoin : plus de 600 visionnements !

La semaine de la recherche 2020 a également été l'occasion d'exposer et de soutenir les projets de recherche de membres de notre corps professoral et de la population étudiante. Déborah Safi, une des assistantes de recherche du $\mathrm{CA}_{A}$ et étudiante au programme de baccalauréat en administration des affaires a, quant à elle, contribué à une exposition Photo-voix, une activité coordonnée par les professeures Chantal White et Stéphanie St-Pierre qui a permis à une vingtaine d'étudiantes et étudiants de développer l'esprit critique d'un enjeu en s'appuyant sur des photos.

En février, $\mathrm{M}^{\mathrm{me}}$ Valérie Caron, responsable du développement à la direction régionale Amériques de l'Agence universitaire de la francophonie (Auf), est venue présenter, au campus de la Pointe-de-l'Église, les différents programmes de l'organisation. L'activité, organisée par le Centre acadien, avec l'appui du bureau du vice-recteur à l'enseignement et à la recherche, a permis de découvrir les opportunités disponibles pour les professionnels de l'enseignement et de la recherche.

\section{Projets et partenariats}

Grâce à une subvention provinciale du Programme provincial de développement d'archives, administré par le ministère des Communautés, de la culture et du patrimoine, nous avons embauché Stéphanie St-Pierre, chargée de cours et chercheuse associée à la chaire CRÉAF (dont le titulaire est Jimmy Thibault), et Yvette Comeau, étudiante à la maîtrise en espace et cultures francophones, à titre d'assistantes aux archives pour une partie de l'année. La subvention a permis d'indexer trois fonds d'archives : ceux de la professeure Susan Knutson, des Eudistes de Charlesbourg et de l'historien Alphonse Deveau, ancien directeur et fondateur du Centre acadien.

À l'automne 2019, le centre s'est engagé à appuyer le projet « Trois siècles de migrations francophones en Amérique du Nord, 1640-1940 », coordonné par Yves Frenette, professeur de l'Université de Saint-Boniface. Le CA est partenaire institutionnel du professeur Clint Bruce, titulaire de la chaire CRÉACT et chercheur associé à ce projet sur les mouvements migratoires. En plus de contribuer à élucider un aspect jusqu'alors méconnu de l'histoire acadienne en Nouvelle-Écosse et partout dans la diaspora, ce partenariat permettra de mettre en valeur les importantes ressources du CA. De nouveaux documents textuels, photographiques et sonores pourront éventuellement s'ajouter à nos collections. 


\section{Expériences de travail}

Grâce à l'obtention de subventions du programme Emploi d'été Canada 2020, le Centre acadien a embauché deux étudiantes de premier cycle de l'Université Sainte-Anne : Ramona Blinn et Carolanne Martel ; elles ont surtout travaillé à la création d'index et d'inventaires qui faciliteront l'accès à nos ressources. Elles ont également contribué au projet sur les migrations, en identifiant des ressources et collections pertinentes à recenser. Nous avons également embauché deux étudiantes de l'Université Sainte-Anne à titre d'assistantes de recherche au cours de l'année universitaire. Au semestre d'automne, Louise d'Alessio-Doucet, récemment diplômée, s'est jointe à notre équipe afin de répondre aux demandes de recherches généalogiques. À l'hiver, ce fut au tour de Déborah Safi ; bien que sa principale tâche fût de traiter les demandes de recherche, elle a également été responsable de tâches liées à l'accessibilité de nos ressources ; elle a créé un index pour une de nos collections de photos et contribué à l'indexation d'archives.

La pandémie et le confinement ont considérablement ralenti nos activités ; nous avons dû annuler les activités publiques prévues pour la saison estivale, mais plusieurs projets et la participation étudiante se poursuivent grâce au télétravail.

Ramona Blinn et CARmen D’Entremont

\section{Centre d'études acadiennes Anselme-Chiasson}

Université de Moncton

Moncton (Nouveau-Brunswick)

E1A 3E9
Téléphone : (506) 858-4085

Télécopieur : (506) 858-4530

Courriel : robert.richard@umoncton.ca

Toile : www.umoncton.ca/umcm-ceaac

\section{Laboratoire de conservation et traitement}

Durant la période d'isolement (Covid-19), Donald C. Rail, administrateur de systèmes informatiques attitré au CÉAAC, en a profité pour migrer tous nos documents numériques vers un nouvel espace de travail en ligne, une suite d'applications de productivité et de collaboration. Privilégié par l'Université de Moncton, ce service augmente notre capacité de stockage, de sauvegarde, d'accès, de partage et de traitement des données. Il facilite également le télétravail.

Diplômé du Département de musique de l'Université de Moncton en 2019, Nicholas Elliott a poursuivi le travail d'analyse et de transcription des mélodies de la collection manuscrite de chansons de l'abbé Pierre-Paul Arsenault (1867-1927). Le programme de subvention Jeunesse Canada au travail dans un établissement du patrimoine (Patrimoine canadien) a défrayé environ $75 \%$ de son salaire. Depuis le début de la Covid-19, Nicholas Elliott a réamorcé ce travail.

\section{Communications, consultations et participation}

En septembre et novembre, Robert Richard, archiviste au CÉAAC, a livré deux communications. Offerte au Symposium sur l'histoire orale du Canada atlantique à l’Université de l'Île-du-Prince-Édouard, la première s'intitulait « Intangible Cultural 